\title{
Acute oral toxicity of squid and cuttlefish ink powder enzyme hydrolysates in Sprague-Dawley rats in accordance with the OECD Test Guideline 425
}

\author{
${ }^{1}$ Ayu Shazwani, Z., ${ }^{2}$ Husnul Azan, T., ${ }^{3}$ Wan Ezumi M.F. and ${ }^{1, *}$ Rabeta, M.S. \\ ${ }^{1}$ Food Technology Division, School of Industrial Technology, Universiti Sains Malaysia, 11800 Penang, \\ Malaysia \\ ${ }^{2}$ Bioprocess Technology Division, School of Industrial Technology, Universiti Sains Malaysia, 11800 \\ Penang, Malaysia \\ ${ }^{3}$ Biomedicine Programme, School of Health Sciences, Health Campus, Universiti Sains Malaysia, 16150 \\ Kubang Kerian, Kelantan, Malaysia
}

\begin{abstract}
Article history:
Received: 31 March 2021

Received in revised form: 24

April 2021

Accepted: 27 April 2021

Available Online: 30 October 2021
\end{abstract}

\section{Keywords:}

Squid ink,

Cuttlefish ink,

Hydrolysate,

Acute oral toxicity,

Histology

DOI:

https://doi.org/10.26656/fr.2017.5(5).219

\begin{abstract}
The objectives of this study were to determine $\mathrm{LD}_{50}$ and establish the safety of ink squid and cuttlefish hydrolysates. In the acute toxicity study, three groups of female rats were randomly assigned. One group served as the control and two groups orally received a single limiting dose $(2000 \mathrm{mg} / \mathrm{kg}$ body weight) of ink hydrolysates. There were no signs of adverse toxicity observed in behavioural patterns, clinical signs, and no significant differences $(p>0.05)$ between the control and treated rats regarding their food and water consumption and body weight for up to 14 days. The histopathological evaluation revealed a normal structure and the absence of noticeable lesions in the vital organs of treated animals. It can be concluded that $\mathrm{LD}_{50}$ value is greater than $2000 \mathrm{mg} / \mathrm{kg}$. The results showed that the squid ink powder enzyme hydrolysate (SIPEHs) and cuttlefish ink powder enzyme hydrolysate (CIPEHs) possess low toxicity, as indicated in the rat model. The preliminary results suggested that it should be further evaluated for long-term use and repeated dose effects to support the safe use of these hydrolysates.
\end{abstract}

\section{Introduction}

Cephalopods, including squid, cuttlefish and octopus, are vital economic resources for global fisheries and the main delicacy in export markets (Kechaou et al., 2015). Food and Agriculture Organization [FAO], (2021) reported that approximately 70-80 thousand tons of Cephalopods are annually caught in Malaysia. Hence, the increased exploitation of squid generates a considerable number of by-products and is eventually discarded, yet they are rich in bioactive nitrogenous compounds. These by-products represent $40 \%$ of total body weight, including head, viscera, ink, skin and bones (Wang et al., 2019). Wang et al. (2019) expressed an interest in comparative studies of melanogenesis in squid ink. Melanogenesis is the reaction of melanin pigment formed by the dark colour of ink produced by tyrosinase, dopachrome rearranging enzyme and peroxidase (Ahamed et al., 2018). Melanin is a melanoprotein containing $10-15 \%$ protein composed of essential and non-essential amino acids in squid ink (Lestari et al., 2019).
Squid ink is also known as a multifunctional bioactive compound that stimulates thromboxane production, kills cancer cells, and elevates leukocyte amount (Sasaki et al., 1997). These ink by-products may act as a potential source of secondary metabolites in a broader range of therapeutic applications as alternative medicine (Hossain et al., 2018). In the past few years, hydrolysed proteins are effectively used in functional foods and protein supplementation due to specific health benefits such as reducing protein allergenicity and enhancing protein digestibility (Rao et al., 2016). Furthermore, squid by-products hydrolysate with improved functional properties is common in the food industry (Jiang et al., 2018).

The previous study reported the oral administration of cuttlefish (Sepia officinalis) ink extract at the high dose $(5000 \mathrm{mg} / \mathrm{kg})$ and a limiting dose $(2000 \mathrm{mg} / \mathrm{kg})$ does not result in death nor any sign of toxicity (Soliman et al., 2014). No mortality or evidence of toxicity is found in rats administered with cuttlefish ink at a limiting dose of $2000 \mathrm{mg} / \mathrm{kg}$ per os (Fahmy and Soliman, 2013; Fahmy et al., 2014). However, scanty information 
is available regarding the hydrolysates of squid ink and cuttlefish ink. The toxicological attempts to systematically screen their bioactive compounds are also rare. Given the possible adverse consequences of enriched peptide formulations, every peptide-based substance must be tested for further clinical studies (Barkia et al., 2020).

Therefore, this study was focusing on acute oral toxicity since this is the first study to our knowledge assessing the short-term effects of squid and cuttlefish ink hydrolysates. The hydrolysates were recently developed, and the toxicity study was adequate to provide crucial basic information on the safe acute doses, the potential acute effects, and target organs of toxicity prior to the repeated dose toxicity study. Following this experiment, the subacute, subchronic, and finally, chronic toxicity studies will also be conducted once the appropriate doses of squid and cuttlefish ink hydrolysates were optimized from this current study.

\section{Materials and methods}

2.1 Collection and preparation of squid ink powder enzyme hydrolysates and cuttlefish ink powder enzyme hydrolysates

Squid (Loligo duvancelii) and cuttlefish (Sepia officinalis) were purchased in Taman Tun Saadon Wet Market near Gelugor, Penang. Freshly obtained squid and cuttlefish were dissected, and the ink gland was manually removed from the viscera. The ink glands were placed in a 30-mesh sieve, pressed, and squeezed out the ink. The inks collected were placed in a plastic container to put into the blast freezer (Corbanese Tv Italy) at $-20^{\circ} \mathrm{C}$ for $3 \mathrm{hrs}$. The frozen ink later was entered the freeze dryer (Labconco laboratory freeze dryer) to drain out the water (lyophilization process) and retain the compound. The freeze-dried ink was ground to a fine powder with an electric grinder. Enzymatic hydrolysis was done according to Kim et al. (2015) with slide modification. The squid ink powder and cuttlefish ink powder were dissolved in buffer solution and hydrolyzed using Alcalase ${ }^{\circledR}$ (from Bacillus licheniformis, $2.972 \mathrm{U} / \mathrm{mL}$ ) and Papain (E.C.3.4.22.2, from Carica papaya, 3000 USP-U/mg) at $3 \%(\mathrm{w} / \mathrm{v})$ enzyme/substrate ratio, respectively. The enzyme treatment was conducted according to the recommended dosage, and the optimum temperature was adjusted for all enzymes used. The hydrolysis reaction was conducted for $4 \mathrm{hrs}$ and terminated by placing the hydrolysates in a boiling-water bath at $85^{\circ} \mathrm{C}$ for 20 mins. After centrifugation (Kubota, 4000, Japan) at 10,000 rpm for $20 \mathrm{mins}$, the supernatant was collected, and freeze-dried. The products were referred to as the corresponding squid ink powder enzyme hydrolysate (SIPEHs) and cuttlefish ink powder enzyme hydrolysate (CIPEHs).

\subsection{Experimental animals}

A total of 15 healthy, nulliparous, and non-pregnant Sprague Dawley female rats (8-10 weeks old), weighing $(200 \pm 30 \mathrm{~g})$ animals used were kept in Animal Research and Service Centre (ARASC), Universiti Sains Malaysia. The rats were housed in polycarbonate cages and were given food ad libitum, had free access to distilled water, and the standard condition room (temperature, $25 \pm 5^{\circ} \mathrm{C}$, relative humidity, $50 \pm 5 \%$, and $12 \mathrm{hrs} \mathrm{light/dark} \mathrm{cycles).}$ The procedures followed Institutional Animal Care and Use Committee (IACUC), USM, and the approval number was USM/IACUC/2019/ (117)/ (986).

\subsubsection{Acute toxicity assay}

In accordance with Test Guidelines 425 of the Organization for Economic Cooperation and Development (OECD), the animals were kept for five days under standard conditions (Organization of Economic Co-operation and Development, 2008). After an acclimation period, the animals were randomly divided into three groups $(n=5)$. Two groups were treated with SIPEHs and CIPEHs, and one group (the control) orally received water only. The rats were fasted overnight prior to dosing but had free access to water. A limit test was performed with $(2000 \mathrm{mg} / \mathrm{kg}$ body weight) of ink hydrolysates, which were administered by oral gavage in a single dose. The food was withheld for 3-4 hrs after dosing, but the water was ad libitum. Throughout the period, the rats were inspected for lethality, general health, and development of any physical abnormalities for $6 \mathrm{hrs}$ after dosing, periodically after $48 \mathrm{hrs}, 7$ days and thereafter up to 14 days. Bodyweight was recorded before dosing and periodically after 3 days, 6 days, 9 days and 14 days. The food and water intake were estimated by subtracting the amount of leftover from the total amount provided and recorded daily. In the end, the animals were euthanized using carbon dioxide $\left(\mathrm{CO}_{2}\right)$ in a chamber and the organs (heart, liver, lung, stomach and kidney) were excised. All individual organs were examined for any lesions and the features of treated and control groups were compared. Then, the organs were preserved in $10 \%$ neutral buffered formalin (NBF) for further histopathology evaluation at the School of Biological Sciences, Universiti Sains Malaysia.

\subsubsection{Histopathological analysis}

All isolated organs were fixed, routinely processed, and embedded in paraffin wax. Paraffin sections $(6 \mu \mathrm{m})$ were cut on glass slides and stained with hematoxylin and eosin (H\&E). The slides were examined under a light microscope, and the magnified images of the 
structure of the tissue were captured.

\subsection{Statistical analysis}

The experimental results were expressed as mean \pm standard error mean (SEM), and the statistically analysed by one-way ANOVA followed by multiple comparisons by Turkey's and $\mathrm{P} \leq 0.05$ were considered as statistically significant for bodyweight and cumulative food intake of rats.

\section{Results and discussion}

\subsection{Acute oral toxicity study of animal models}

In the current testing, the limit dose of $(2000 \mathrm{mg} / \mathrm{kg}$ body weight) of SIPEHs and CIPEHs did not cause any sign of toxicity during the 14 days of the experiment. The regular observation of animals showed nonoccurrence signs of distress and no observable symptoms in general conditions in both treated and control groups that were recorded in Table 1. All animals were healthy and showed no changes in physical appearances, such as the skin, fur, eyes, mucous membranes, and respiratory system. Unnoticeable changes were observed in somatomotor activity and behavioural patterns. Tremors, diarrhoea, and coma did not occur in any of the animals. Therefore, we infer that the oral $\mathrm{LD}_{50}$ value was higher than $2000 \mathrm{mg} / \mathrm{kg}$. In principle, the limit test approach recommends the classification of sample tasted based on the estimated dose level at which the animals are expected to survive but not intended to establish a precise $\mathrm{LD}_{50}$ value (Roopashree et al., 2009). The $\mathrm{LD}_{50}$ is a statistically derived single dose of a substance that can cause death in $50 \%$ of animals when administered orally (Organisation of Economic Co-operation and Development, 2001).

Substantial weight loss is typically a key indicator of animal welfare deterioration (Schilter et al., 2003). Instead, the current study revealed no weight loss. There

$\mathrm{C}=$ Control, $\mathrm{SP}=$ SIPEHs, $\mathrm{CP}=$ CIPEHs, $\mathrm{N}=$ Normal, Present $(+)$, Absent $(-)$ were no significant $(\mathrm{p}>0.05)$ differences in the body weight changes between control, and both SIPEHs and CIPEHs treated groups (Figure 1). All rats in each group continued to gain weight throughout the experimental periods. The accumulated weight in rats revealed that the administration of crude extracts did not affect the growth, did not suppress appetite and exerted no harmful effects on the animals. Moreover, in Figure 2 and Figure 3 , data intake showed increased consumption of food and water for control, SIPEHs, and CIPEHs tested groups. The result suggested no disruption in the metabolism of carbohydrates, protein, or lipids because these nutrients play a major role in different physiological functions of the body (Saleem et al., 2017).

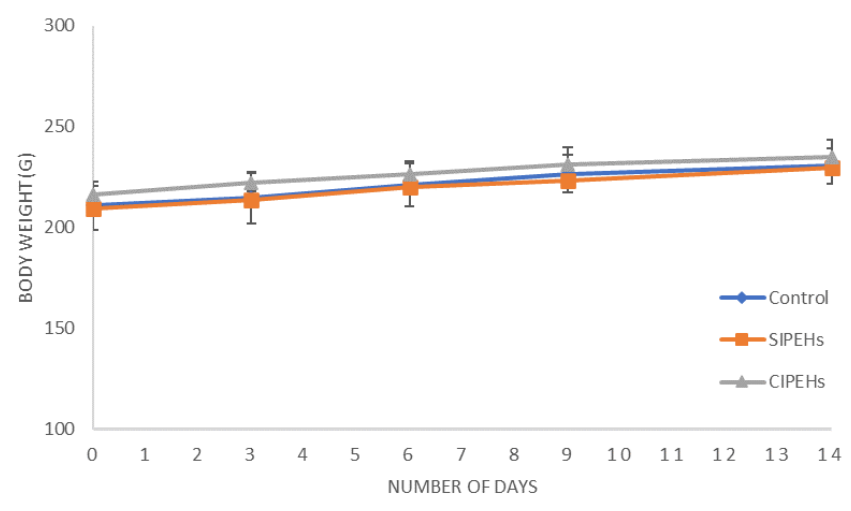

Figure 1. Bodyweight of control, SIPEHs and CIPEHs groups in the acute toxicity (for 14 days) studies. Data indicate mean $\pm \mathrm{SD}, \mathrm{n}=5$.

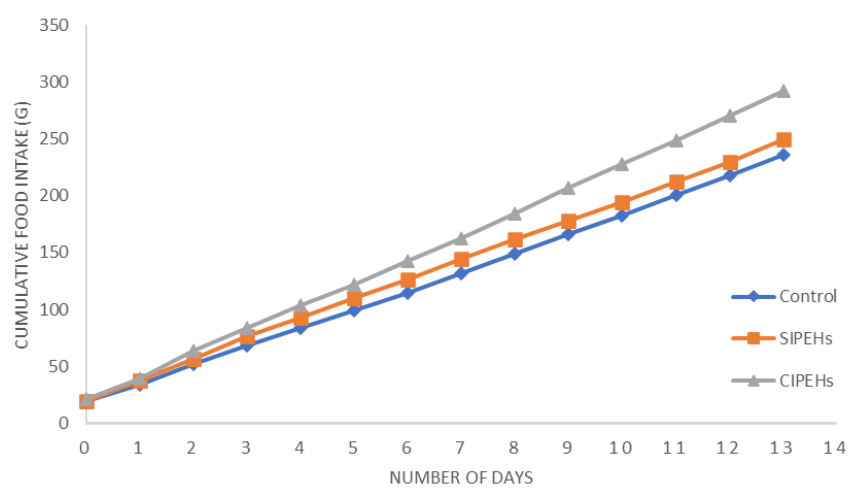

Figure 2. Cumulative food intake of the rats assigned to different groups (control, SIPEHs, and CIPEHs) 


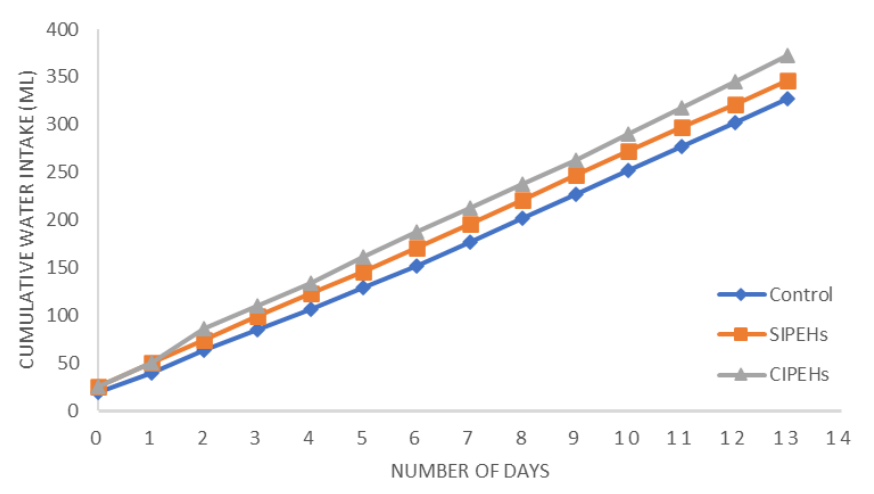

Figure 3. Cumulative water intake of the rats assigned to different groups (control, SIPEHs, and CIPEHs)

\subsection{Gross histopathology findings}

No gross pathological lesions, noticeable alterations were observed, and no differences in systemic organs were exhibited by the groups treated with SIPEHs and CIPEHs compared with the control group (Figure 4). The structure or arrangement of each organ in the extracttreated groups was more or less identical to that in the control group. These data provide useful knowledge regarding the lack of substance-related symptoms, but they remain insufficient for adequately representing the non-toxicity of squid and cuttlefish ink hydrolysates. The integration of histopathological findings would provide an improved understanding of the adverse effects. The organs such as the liver, kidney, heart, lung, and spleen are major target areas of any toxic substance metabolically (Auletta, 1995).

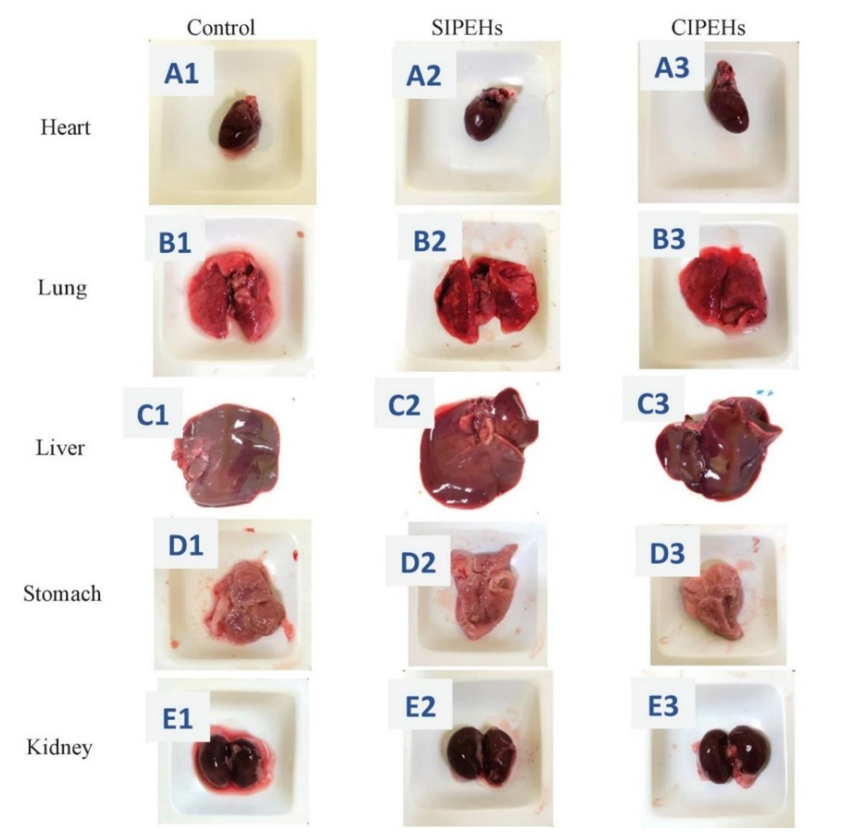

Figure 4. Gross observation of systemic organs: heart (A1, A2, and $\mathrm{A} 3)$, lung $(\mathrm{B} 1, \mathrm{~B} 2$, and $\mathrm{B} 3)$, liver $(\mathrm{C} 1, \mathrm{C} 2$, and $\mathrm{C} 3)$, stomach (D1, D2, and D3), kidney (E1, E2, and E3) from control, SIPEHs and CIPEHs test groups. There were no gross abnormalities and major visual differences observed in all organs. The colour, size, shape, and texture looked comparable with those of the control group.
The autopsy in Figure 5 showed no cell degradation or any unfavourable effects and any differences viewed in animal organs in SIPEHs and CIPEHs treated compared with the control group. The current results for all test groups indicated that the heart showed normal architecture of the myocardium, the lung had a normal alveolar structure, and the liver presented a normal architecture with no evidence of lesion or necrosis was obtained. In addition, the kidney had no trace of glomerular damage or lumen casts and normal structures without any microscopic changes in stomach tissue. Moreover, no evidence of lesion caused by the toxicity of the extracts was observed in the organs. This result means that the dose of SIPEHs and CIPEHs extracts did not affect or change the patterns. The rats did not develop any illness, and only a negligible toxicity level was noted. According to the Globally Harmonized System of classification, chemicals can be divided into five groups based on their $\mathrm{LD}_{50}$ value (Occupational Safety and Health Administration [OSHA], 2013). The crude extracts of SIPEHs and CIPEHs belong to Group 5 $\left(\mathrm{LD}_{50}>2000 \mathrm{mg} / \mathrm{kg}\right)$, a low-toxicity class.

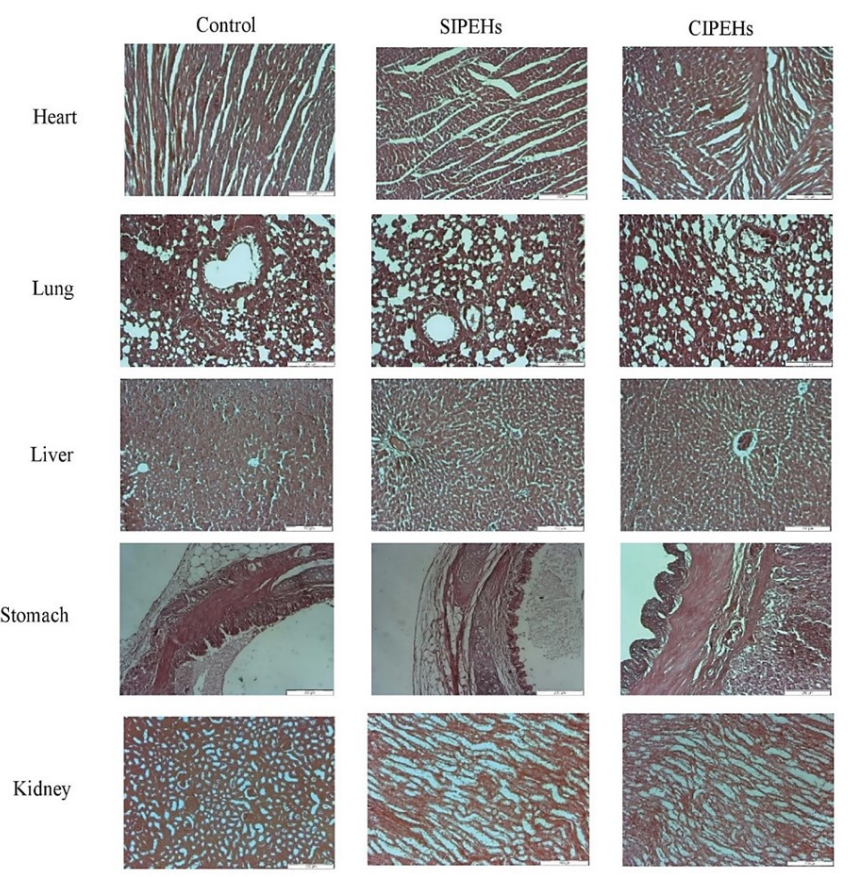

Figure 5. Photomicrographs of hematoxylin eosin-stained systemic organ sections (100X) from the heart, lung, liver, stomach, and kidney of control, SIPEHs and CIPEHs treated rats at a limit dose $(2000 \mathrm{mg} / \mathrm{kg}$ B.W. $)$. There were no indications of toxicity in all visceral organs examined. Their cellular arrangements were normal without major morphological changes and looked comparable with those of the control group. It is important to note that the differences in the staining colour of the slides were due to technical imperfection including staining-batch variations.

Generally, in vivo assays are likely to provide an early indication of the toxicity of a compound because the use of in vitro cytotoxicity methods could result in limited information (Syahmi et al., 2010). Acute oral 
toxicity refers to the adverse effects that occur after oral administration of a single dose or multiple doses within $24 \mathrm{hrs}$ (Organisation of Economic Co-operation and Development, 2001). Based on the literature, oral administration is the most effective and widely used to evaluate acute toxicity. Although absorption may be slow, this method is inexpensive and reasonably painless for animals (Syahmi et al., 2010). Further studies on biochemical and haematological analysis are suggested to be examined when evaluating the toxicity of hydrolysates. In terms of biological parameters, including serum biomarkers, measurement is used to assess body status. For example, liver damage caused by hepatotoxic drugs can be elevated in ALT, AST and total proteins levels (Saleem et al., 2017). Besides, haematological parameters are sensitive markers in the changes of physiological response to environmental pollutants or toxic stress in animals (Jain et al., 2009).

\section{Conclusion}

In conclusion, this acute oral toxicity study of SIPEHs and CIPEHs does not cause any apparent in vivo toxicity in an animal model. No death or signs of toxicity were observed in rats treated with extract at dose 2000 $\mathrm{mg} / \mathrm{kg}$, thus proving the ink hydrolysates possesses low toxicity. Neither gross abnormalities nor histopathological changes were noted in both control and treated groups. However, further research is suggested to reach more specific conclusions about the safety of the high dose of hydrolysates for long periods.

\section{Conflict of interest}

The authors declare no conflict of interest.

\section{Acknowledgements}

This study was financially supported by the Fundamental Research Grant Scheme (FRGS) with grant number 203/PTEKIND/6711526 from Ministry of Higher Education (MOHE) Malaysia and Bridging Grant with grant number 6316287 obtained from Universiti Sains Malaysia. The financial support of Graduate Assistance (GA) Scheme from Universiti Sains Malaysia for author Ayu Shazwani Z was gratefully acknowledged. We would like to express my heartfelt gratitude to Dr. Isma Suzyta Ismail as a Veterinary Officer in this study.

\section{References}

Ahamed, A.R.G.B., Hossain, M.P., Antora, R.A. and Rabeta M.S. (2018). Physical and functional properties of Indian squid (Loligo duvaucelii) and cuttlefish (Sepia latimanus) ink powder. Food
Research, 2(4), 314-319. https://doi.org/https:// doi.org/10.26656/fr.2017.2(4).070

Auletta, C.S. (1995). Acute, subchronic and chronic toxicology. Handbook of Toxicology, p. 51-162. USA: CRC Press.

Barkia, I., Bouaziz, H.K., Boudawara, T.S., Aleya, L., Gargouri, A.F. and Saari, N. (2020). Acute oral toxicity study on Wistar rats fed microalgal protein hydrolysates from Bellerochea malleus. Environmental Science and Pollution Research, 27 (16), 19087-19094. https://doi.org/10.1007/s11356018-4007-6

Fahmy, R. and Soliman, M. (2013). In vitro antioxidant, analgesic and cytotoxic activities of Sepia officinalis ink and Coelatura aegyptiaca extracts. African Journal of Pharmacy and Pharmacology, 7(22), 1512-1522. https://doi.org/10.5897/AJPP2013.3564

Fahmy, S.R., Ali, E.M. and Ahmed, N.S. (2014). Therapeutic effect of Sepia ink extract against invasive pulmonary aspergillosis in mice. The Journal of Basic and Applied Zoology, 67(5), 196204. https://doi.org/10.1016/j.jobaz.2014.08.001

Food and Agriculture Organization (FAO) (2021). Fishery and Aquaculture Country Profiles, Malaysia (2009). Country Profile Fact Sheets. Updated 1 February 2009. Retrieved on January 29, 2020 from FAO Website: http://www.fao.org/fishery/

Hossain, M.P., Rabeta, M.S. and Husnul Azan, T. (2018). Medicinal and therapeutic properties of cephalopod ink: A short review. Food Research, 3 (3), 188-198. https://doi.org/10.26656/fr.2017.3 (3). 201

Jain, N., Sharma, P., Sharma, N. and Joshi, S.C. (2009). Haemato-biochemical profile following sub acute toxicity of malathion in male albino rats. Avicenna Journal of Phytomedicine, 2, 500-506.

Jiang, W., Liu, Y., Yang, X., Wang, P., Hu, S. and Li, J. (2018). Recovery of Proteins from Squid ByProducts with Enzymatic Hydrolysis and Increasing the Hydrolysate's Bioactivity by Maillard Reaction. Journal of Aquatic Food Product Technology, 27(8), 900-911. doi.org/10.1080/10498850.2018.1508104

https://

Kechaou, E.S., Bergé, J.P., Jaouen, P. and Ben Amar, R. (2015). Optimization of common cuttlefish (Sepia officinalis) protein hydrolysate using pepsin by response surface methodology. Journal of Aquatic Food Product Technology, 24(3), 270-282. https:// doi.org/10.1080/10498850.2013.773116

Kim, N., Son, H., Maeng, J.S., Cho, Y.J. and Kim, C.T. (2015). Enzymatic hydrolysis of anchovy fine powder at high and ambient pressure and 
characterization of the hydrolyzates. Journal of the Science of Food and Agriculture, 96(3), 970-978. https://doi.org/10.1271/bbb.70.729

Lestari, R., Junianto, J., Mulyani, Y. and Pratama, R.I. (2019). Addition of squid ink (Loligo vulgaris Lamarck, 1798) on tortilla chips to favorite levels. World News of Natural Sciences, 25, 155167.

Occupational Safety and Health Administration (OSHA) (2013). Globally harmonized system of classification and labelling of chemicals (GHS). Geneva, Switzerland: United Nations Economic Commission for Europe (UNECE).

Organization of Economic Co-operation and Development (2001). The OECD guideline for testing of chemical: 407 repeated dose oral toxicityrodent: 28-day or 14-day study. Paris, France: OECD.

Organization of Economic Co-operation and Development (2008). Guidelines for Testing of Chemicals: Guideline 425: Acute Oral Toxicity. Paris, France: OECD.

Saleem, U., Amin, S., Ahmad, B., Azeem, H., Anwar, F. and Mary, S. (2017). Acute oral toxicity evaluation of aqueous ethanolic extract of Saccharum munja Roxb. roots in albino mice as per OECD 425 TG. Toxicology Reports, 4, 580-585. https:// doi.org/10.1016/j.toxrep.2017.10.005

Sasaki, J.I., Ishita, K., Takaya, Y., Uchisawa, H. and Matsue, H. (1997). Anti-tumor activity of squid ink. Journal of Nutritional Science and Vitaminology, 43 (4), 455-461.

Schilter, B., Andersson, C., Anton, R., Constable, A., Kleiner, J., O'Brien, J., Renwick, A.G., Korver, O., Smit, F. and Walker, R. (2003). Guidance for the safety assessment of botanicals and botanical preparations for use in food and food supplements. Food and Chemical Toxicology, 41(12), 1625-1649. https://doi.org/10.1016/S0278-6915(03)00221-7

Soliman, A.M., Marie, M.A.S., Saleh, H.M. and Mohamed, A.S. (2014). Assessment of sepia ink extract role against the kidney dysfunction induced by bile duct ligation. The Journal of Basic and Applied Zoology, 67(5), 173-181. https:// doi.org/10.1016/j.jobaz.2014.04.001

Syahmi, A.R.M., Vijayarathna, S., Sasidharan, S., Latha, L.Y., Kwan, Y.P., Lau, YL., Shin, L.N. and Chen, Y. (2010). Acute oral toxicity and brine shrimp lethality of Elaeis guineensis Jacq. (oil palm leaf) methanol extract. Molecules, 15(11), 8111-8121. https:// doi.org/10.3390/molecules 15118111

Rao, Q., Klaassen Kamdar, A. and Labuza, T.P. (2016).
Storage Stability of Food Protein Hydrolysates-A Review. Critical Reviews in Food Science and Nutrition, 56(7), 1169-1192. https:// doi.org/10.1080/10408398.2012.758085

Roopashree, T.S., Dang, R., Shobha Rani, R.H. and Narendra, C. (2009). Acute oral toxicity studies of antipsoriatic herbal mixture comprising of aqueous extracts of Calendula officinalis, Momordica charantia, Cassia tora and Azadirachta indica seed oil. Thai Journal of Pharmaceutical Sciences, 33(2), 74-83.

Wang, C.H., Doan, C.T., Nguyen, V.B., Nguyen, A.D. and Wang, S.L. (2019). Reclamation of fishery processing waste: A mini review. Molecules, 24(12), 2234. https://doi.org/10.3390/molecules24122234 